\title{
ORTHOMODULAR POSETS FROM SESQUILINEAR FORMS
}

\author{
ROBERT PIZIAK \\ (Received 28th April 1971) \\ Communicated by P. D. Finch
}

In this paper, we show how to generate orthomodular posets from sesquilinear forms on a vector space.

Let $E$ be a vector space over the division ring $k$. A binary relation $\perp$ on $E$ is called a linear orthogonality relation provided

(1) $x \perp y$ iff $y \perp x$, and

(2) for each $x$ in $E,\{x\}^{\perp}=\{y \mid y \perp x\}$ is a linear subspace of $E$.

For a subset $M$ of $E$ we define the orthogonal of $M$ by

$$
M^{\perp}=\{y \mid y \perp m \text { for all } m \text { in } M\} .
$$

Also we let $[M]$ denote the linear span of $M$ in $E$.

The first lemma is trivial.

LeMma 1. For $M, M_{i}$, and $N$ subsets of $E$, we have

(1) $M \subseteq M^{\perp \perp}$

(2) $M \subseteq N$ implies $N^{\perp} \subseteq M^{\perp}$

(3) $M^{\perp}=M^{\perp \perp \perp}$

(4) $\left(\cup M_{i}\right)^{\perp}=\cap M_{i}^{\perp}$

(5) $M^{\perp}$ is a subspace of $E$

(6) $M^{\perp}=[M]^{\perp}$ so in particular if $x \perp y$ and $x \perp z$ then $x \perp y+z$ and $x \perp \alpha y$ for all $\alpha$ in $k$.

(7) $(0)^{\perp}=E$ and $E=E^{\perp \perp}$

Note that $M \mapsto M^{\perp \perp}$ is a closure operator on the lattice of all subspaces of $E$.

Let $\perp$ be a linear orthogonality relation on $E$. We say $\perp$ is nondegenerate when $E^{\perp}=(0)$. In this case we call $(E, \perp)$ a linear orthogonality space.

Call a subspace $M$ of $E$ orthogonally closed or $\perp$-closed if $M=M^{\perp \perp}$. Let $P_{c}(E, \perp)$ denote the set of all $\perp$-closed subspaces of $E$ ordered by inclusion. Using well known generalities on closure operators we see that $P_{c}(E, \perp)$ is a comlete involution lattice with zero (0) and unit $E$. Also for $M_{i}$ in $P_{c}(E, \perp)$ we have 


$$
\inf \left(M_{i}\right)=\cap M_{i} \text { and } \sup \left(M_{i}\right)=\left[\cup M_{i}\right]^{\perp \perp} .
$$

Call a vector $x$ in $E$ isotropic if $x \perp x$ and anisotropic otherwise. For a subspace $F$ of $E$, define the radical of $F$ by $\operatorname{rad}(F)=F \cap F^{\perp}$. Say that $F$ is semisimple provided $\operatorname{rad}(F)=(0)$. Let $P_{s s}(E, \perp)$ denote the set of all semisimple subspaces of $E$ ordered by inclusion.

It can be shown that the orthogonal of a semisimple subspace need not be semisimple. However,

$$
P_{s s}(E, \perp) \cap P_{c}(E, \perp)
$$

is easily seen to be an orthocomplemented poset under the natural involution $F \mapsto F^{\perp}$. It need not though be orthomodular.

LEMMA 2. Let $\left\{F_{i}\right\}$ be an orthogonal family of linear subspaces of $E$ (i.e. $F_{i} \subseteq F_{j}^{\perp}$ for $i \neq j$ ). Let $F$ be the smallest subspace of $E$ containing all the $F_{i}$. We write $F=\Sigma F_{i}$. Then $\operatorname{rad}(F)=\Sigma \operatorname{rad}\left(F_{i}\right)$.

Proof. First $\operatorname{rad}(F)=F \cap F^{\perp}=F \cap\left(\cap F_{i}^{\perp}\right)=\cap\left(F \cap F_{i}^{\perp}\right)$. For each fixed $j$ and for any $i$ we have

$$
\operatorname{rad}\left(F_{j}\right)=F_{j} \cap F_{j}^{\perp} \subseteq F \cap F_{i}^{\perp} .
$$

Hence for each $j, \operatorname{rad}\left(F_{j}\right) \subseteq \operatorname{rad}(F)$ so $\sum \operatorname{rad}\left(F_{i}\right) \subseteq \operatorname{rad}(F)$.

Conversely, suppose $x$ is in $\operatorname{rad}(F)$. Since $x$ is in $F$ we can write $x$ as a finitely nonzero sum $x=\sum x_{i}$ with $x_{i}$ in $F_{i}$. For each $j$,

$$
x_{j}=x-\sum_{i \neq j} x_{i}
$$

Since $x$ is in $\operatorname{rad}(F)$ then $x$ is in $F_{j}^{\perp}$. Since the family $\left\{F_{i}\right\}$ is orthogonal, each $x_{i}$ with $i \neq j$ also belongs to $F_{j}^{\perp}$. Thus $x_{j}$ is in $F_{j}^{\perp}$. It follows each $x_{j}$ is in $\operatorname{rad}\left(F_{j}\right)$. Thus $x$ is in $\Sigma \operatorname{rad}\left(F_{i}\right)$.

COROLlaRY 3. If $\left\{F_{i}\right\}$ is an orthogonal family of semisimple subspaces of $E$, the join exists in $P_{s s}(E, \perp)$ and in fact the join is the orthogonal direct sum of the $F_{i}$.

Next we have a technical lemma.

Lemma 4. Let $F$ and $G$ be linear subspaces of $E$. Suppose $F \subseteq G$ and $G \subseteq F+F^{\perp}$. Let $G$ be semisimple. Then $G \cap F^{\perp}$ is semisimple.

PROOF. If $G \cap F^{\perp}$ were not semisimple, we would have a vector $w$ different from zero with $w$ belonging to

$$
\operatorname{rad}\left(G \cap F^{\perp}\right)=\left(G \cap F^{\perp}\right) \cap\left(G \cap F^{\perp}\right)^{\perp} .
$$

Since $G$ is semisimple and $w$ is in $G$ we cannot have $w$ in $G^{\perp}$. Thus there 
is a vector $y$ in $G$ such that $y$ fails to be orthogonal to $w$. Since $G$ is contained in in $F+F^{\perp}$ and $F$ is contained in $G$ we see

$$
G=F+\left(F^{\perp} \cap G\right) \text {. }
$$

Hence we can write $y=u+x$ where $u$ belongs to $F$ and $x$ is in $F^{\perp} \cap G$ Since $w$ is in $F^{\perp}$ and $w$ is in $\left(F^{\perp} \cap G\right)^{\perp}$, then $w$ is orthogonal to $y$, a contradiction.

A subspace $F$ of the linear orthogonality space $(E, \perp)$ is called splitting if $E=F+F^{\perp}$. Let $P_{s}(E, \perp)$ denote the set of all splitting subspaces of $E$ again ordered by inclusion.

The next lemma is straightforward and we omit the proof.

LeMmA 5. (1) (0) and $E$ are splitting subspaces

(2) if $F$ is in $P_{s}(E, \perp)$ then so is $F^{\perp}$

(3) every splitting subspace is closed and semisimple

(4) $P_{s}(E, \perp)$ is an orthocomplemented poset under the inovolution $F \rightarrow F^{\perp}$.

The next lemma establishes the first crucial property of an orthomodular poset.

LemMa 6. Finite orthogonal joins exist in $P_{s}(E, \perp)$.

Proof. Let $e$ be any vector in $E$. Let $F$ and $G$ be in $P_{s}(E, \perp)$ with $F \subseteq G^{\perp}$. We claim

$$
F+G=F \oplus G
$$

is in $P_{s}(E, \perp)$. First $e=w+w_{1}$ with $w$ in $G$ and $w_{1}$ in $G^{\perp}$ and $e=v+v_{1}$ with $v$ in $F$ and $v_{1}$ in $F^{\perp}$. Clearly

$$
e=(v+w)+x
$$

where $x=e-v-w, v_{1}-w=w_{1}-v$. Since $v_{1}$ is in $F^{\perp}$ and $w$ is in $G$ then $x$ is in $F^{\perp}$. Similarly, $x$ is in $G^{\perp}$. Thus $e$ is in

$$
(F+G)+\left(F^{\perp} \cap G^{\perp}\right)=(F+G)+(F+G)^{\perp} .
$$

Hence $E=(F+G)+(F+G)^{\perp}$

We now come to the main result.

THEOREM 7. Let $(E, \perp)$ be a linear orthogonality space. Then $P_{s}(E, \perp)$ is an orthomodular poset.

Proof. We have already that $P_{s}(E, \perp)$ is an orthocomplemented poset with zero (0) and unit $E$ under the involution $F \mapsto F^{\perp}$. Orthogonal joins are just orthogonal direct sums. It suffices then to show the orthomodular identity. Let $F$ and $G$ be spliting subspaces with $F \subseteq G$. Then $G^{\perp} \leqq F^{\perp}$ so $F$ is orthogonal to $G^{\perp}$ so $F \vee G^{\perp}=F+G^{\perp}$. Thus 


$$
\left(F \vee G^{\perp}\right)^{\perp}=\left(F+G^{\perp}\right)^{\perp}=F^{\perp} \cap G .
$$

Now $G=G \cap E=G \cap\left(F+F^{\perp}\right)=F+\left(G \cap F^{\perp}\right)=F \vee\left(F \vee G^{\perp}\right)^{\perp}$ which completes the proof.

Note if $E$ is finite dimensional, $P_{s}(E, \perp)$ is necessarily an atomic orthomodular poset. Also note that linear orthogonality relations exist in great abundance. Let $E$ be any vector space. Let $\Phi$ be a $\theta$-sesquilinear nondegenrate orthosymmetric from on $E$. For $x$ and $y$ in $E$, define $x \perp y$ by $\Phi(x, y)=0$. Then $(E, \perp)=(E, \Phi)$ is a linear orthogonality space. Call such a quadratic space. We have characterized which linear orthogonality spaces are quadratic spaces elsewhere. For a quadratic space $(E, \Phi)$ it can also be shown that $P_{s}(E, \Phi)$ is an ample atomic orthomodular poset with the ortho-covering and ortho-exchange properties.

A crucial problem is to determine when $P_{s}(E, \Phi)$ is a lattice. The next theorem provides and important partial answer. We are indebted to $H$. R. Fischer for the proof.

THEOREM 8. Let $(E, \Phi)$ be a quadratic space of dimension at least 4 over a field of characteristic different from two. Suppose not every vector of $E$ is isotropic. If $P_{s}(E, \Phi)$ is a lattice then $\Phi$ admits no non-zero isotropic vectors.

\section{Proof.}

Suppose on the contrary that $\Phi$ admits a nonzero isotropic vector. Since every nondegenerate space of dimension at least 4 contains a four dimensional semisimple subspace, it suffices to consider the case where the dimension of $E$ equals 4 and show that $E$ contains two distinct three dimensional semisimple subspaces whose intersection is a degenerate plane, but not totally isotropic (i.e. with radical properly contained in this plane, thus of dimension one).

The proof proceeds as follows: we shall construct in $E$ a plane $[x, y]$ such that $x \perp x, y \perp y$, and $x \perp y$. Then we shall find two distinct three dimensional semisimple spaces $F$ and $G$ in $E$ such that $F \cap G=[x, y]$. Once this is done, it is clear that $F$ and $G$ do not possess any infimum in $P_{s}(E, \Phi) ;[y]$ and $[x+y]$ are distinct noncomparable lower bounds of $F$ and $G$ in $P_{s}(E, \Phi)$.

The construction is as follows. Choose any nonzero $x$ in $E$ such that $x \perp x$. Then $[x]^{\perp}$ is a subspace of dimension three. Therefore it cannot be totally isotropic. Now choose anisotropic $y$ in $[x]^{\perp}$. Then $[x, y]$ is the required plane. It is degenerate with $\operatorname{rad}([x, y])=[x]$.

Next $[y]^{\perp}$ is three dimensional and semisimple. Since $x$ is in $[y]^{\perp}$ there exists $a$ in $[y]$ such that $x \not \subset a$. If $a$ is anisotropic, let $z=a$. If $a$ is isotropic, let $z=a+x$. This will be anisotropic and still not orthogonal to $x$. In either case we have an anisotropic $z$ in $[y]^{\perp}$ such that $x \not 1 z$. From this it follows that $[x, y, z]$ is semisimple; its radical is properly contained in $[x]$ whence is $(0)$.

The three dimensional space $F=[x, y, z]$ is also spanned by $x, x+y$, and $z$. 
Since $x+y$ is anisotropic $[x+y]^{\perp}$ is semisimple. Hence, $x$ being in $[x+y]$ there is an anisotropic $u$ in $[x+y]^{\perp}$ such that $x \not \perp u$. Then $[x, x+y, u]=[x, y, u]$ is again semisimple and of dimension three.

We now have two cases:

case $a: u$ is not in $[x, y, z]$. Then we put $G=[x, y, u]$ and get $F \cap G=[x, y]$.

case $b: u$ is in $[x, y, z]$. By construction, $\{x, x+y, u\}$ is a linearly ind $\epsilon \in \in$ C $\mathrm{C}^{\prime} \in \mathrm{nt}$ subset of $F$ and hence is a basis of $F$. In particular, $[x, x+y, u]^{\perp}=$ $[u]^{\perp} \cap[x]^{\perp}=[x+y]^{\perp}$ is one dimensional semisimple, i.e. is spanned by an anisotropic vector $a$. Now $u$ is in $[x+y]^{\perp}=[u] \oplus M, M$ of dimension two and semisimple. Note that $M=[u]^{\perp} \cap[x+y]^{\perp}$. Also $[a]=[u]^{\perp} \cap[x]^{\perp} \cap[x+y]^{\perp}$ $=[x]^{\perp} \cap M$. Thus there exists an anisotropic vector $w$ in $M$ such that $a \perp w$. Clearly then $w$ is not in $[x]^{\perp}$ but $w$ is in $[x+y]^{\perp}$. In this case we put $G$ $=[x, x+y, w]=[x, y, w]$. Again we have $F \cap G=[x, y]$ both $F$ and $G$ semisimple of dimension three. This completes the proof.

We remark that if the dimension of $E$ does not exceed 3, then $P_{s}(E, \Phi)$ is a lattice simply because there is not enough height for things to go wrong. If in the above theorem, the dimension of $E$ is finite and $\Phi$ admits no nonzero isotropic vectors, then $P_{s}(E, \Phi)=P_{s s}(E, \Phi)$ is the lattice of all subspaces of $E$. Also in particular we note that if $\Phi$ is the Minkowski metric fo space-time, then $P_{s}\left(\mathbb{R}^{4}, \Phi\right)$ is an orthomodular poset that is not an orthomodular lattice.

We close with some open questions:

QUESTION 1. What about the converse of Theorem 8 ?

QUESTION 2. What is the cut completion of $P_{s}(E, \perp)$ ?

\section{Acknowledgement}

The author wishes to express his thanks to D. J. Foulis for his help.

\section{References}

[1] N. Bourbaki, Livre II Algèbre, Chapître IX, Forme Sesquilinéaire et Formes Quadratiques (Hermann, Paris, VI, 1959),

[2] O. T. O'Maera, Introduction to Quadratic Forms (Springer Verlag, Band 117, (1963)).

[3] J. C. T. Pool, Simultaneous Observability and the Logic of Quantum Mechanics, (Ph. D. Thesis, Iowa University, 1963).

[4] R. Piziak, An Algebraic Generalization of Hilbert Space Geometry (Ph. D. Thesis, Massachusetts 1969).

[5] A. Ramsay, 'A Theorem on Two Commuting Observables' J. Math. and Mech. 15 (1966). 227-234.

Department of Mathematics

University of Florida

205 Walker Hall, Gainesville

Florida 32601 U. S. A. 